Eixo Temático: Biologia Aplicada

\title{
ET-09-021 \\ ANÁLISE PRELIMINAR DA COMUNIDADE PLANCTÔNICA DO ESTUÁRIO DO RIO SAUÍPE (ENTRE RIOS, BAHIA - BRASIL)
}

Milena Evangelista Santos¹, Milena Gonçalves da Silva', Maria Dolores Ribeiro Orge², Rogenaldo de Brito Chagas ${ }^{3}$

${ }^{1}$ Graduandas em Licenciatura em Ciências Biológicas, Universidade do Estado da Bahia UNEB.

2Professora Adjunto da Universidade do Estado da Bahia (UNEB), Depto. de Ciências Exatas e da Terra (Campus II, Alagoinhas).

${ }^{3}$ Professor da Universidade do Estado da Bahia (UNEB), Depto. de Ciências Exatas e da Terra (Campus II, Alagoinhas)

http://dx.doi.org/10.21472/congrebio2016.et-09-021

\section{RESUMO}

Este trabalho teve por finalidade realizar o estudo da comunidade planctônica do Estuário do Rio Sauípe, localizado no Distrito de Porto de Sauípe, Município de Entre Rios (Bahia - Brasil), possuindo cunho informativo, já que existem poucos estudos no Litoral Norte deste estado envolvendo os dois grupos, fitoplâncton e zooplâncton. Para a coleta do material foram realizados arrastos superficiais com o auxílio de uma rede de plâncton cônica com malha de $60 \mu \mathrm{m}$ com $30 \mathrm{~cm}$ de abertura na boca e $68 \mathrm{~cm}$ de comprimento, com copo rosqueável de 20 cm. Logo após os arrastos, o material foi acondicionado em uma garrafa e para a conservação dos microrganismos foi acrescentado Solução de Transeau. O material foi filtrado com papel filtro e o concentrado foi analisado em microscópio óptico, para após serem calculadas as frequências absoluta e relativa. Para o estuário foi realizado a coleta de um único ponto amostral, resultando na identificação de 367 indivíduos, distribuídos em 19 taxa e dois Reinos, com os indivíduos pertencentes ao Reino Protozoa, possuindo maior representatividade dentre os demais taxa analisados, com uma percentagem de $84,2 \%$. Os resultados nos conferem a necessidade da continuação das pesquisas exploratórias no estuário do rio Sauípe para a avaliação mais precisa sobre a qualidade do ecossistema, visto que neste estudo preliminar foram encontrados representantes dos protozoários.

Palavras-chave: Fitoplâncton; Zooplâncton; Rio Sauípe.

\section{INTRODUÇÃO}

Os estuários são corpos de águas costeiras, com conexão direta com o mar aberto, que são diluídas pela água epicontinental proveniente da drenagem terrestre. Os ecossistemas estuarinos são regiões importantes, que exercem atividades biológicas fundamentais, já que são altamente produtivos. Caracterizam-se por ambientes dinâmicos, com constantes modificações nos fatores abióticos e bióticos, que influenciam na diversidade de indivíduos que podem ser encontrados. A biota estuarina é derivada dos ambientes terrestre, marinho e de água doce, além de uma fauna e flora autóctones.

O plâncton (fitoplâncton e zooplâncton) é a base da teia alimentar e podem influenciar nos processos ecológicos de ciclagem de nutrientes e na produção biológica. O fitoplâncton é o principal produtor primário dos oceanos, constituído por microalgas unicelulares (euglenófitas, dinófitas e cianófitas), realizando atividade fotossintética na zona eufótica, sendo importantes 
pois são responsáveis pela totalidade das teias alimentares marinhas (YONEDA, 1999). Já os zooplânctons correspondem aos metazoários microscópicos, livre-natantes ou suspensos (rotíferos, microcustáceos, celenterados, platelmintos e protozoários) (TUNDISI, 2008). Podem ser divididos em dois grupos básicos: holoplâncton, que inclui aqueles que passam todo o ciclo de vida no plâncton; e o meroplâncton, que engloba os ovos, larvas e juvenis, cujos adultos fazem parte das comunidades bênticas ou nectônicas (YONEDA, 1999).

Atualmente já existem pesquisas sobre a comunidade planctônica no Litoral Norte do Estado da Bahia (Brasil), incluindo o Porto de Sauípe, como se observa nos trabalhos de Oliveira et al. (2011) e Sousa (2013). Mesmo assim, os estudos ainda são escassos e alguns restritos a gêneros, o que acarreta a necessidade de estudos mais aprofundados sobre a comunidade e ecologia de fito e zooplânctons ocorrentes no estuário do município de Porto de Sauípe.

\section{OBJETIVOS}

O objetivo deste trabalho é fazer um levantamento preliminar da comunidade planctônica do Estuário do Rio Sauípe, Entre Rios/Ba.

\section{METODOLOGIA}

A área de estudo está localizada no estuário do rio Sauípe ( $12^{\circ} 24^{\prime} \mathrm{S}$ e $\left.37^{\circ} 53^{\text {’ W }}\right)$, situada no distrito de Porto de Sauípe, município de Entre Rios, pertencente ao Litoral Norte do Estado da Bahia (Brasil) e distante $80 \mathrm{~km}$ da capital Salvador (Figura 1). Este ecossistema está associado a manguezais em ambas margens, que são utilizados pela comunidade local para as atividades pesqueiras e turismo. O Distrito de Porto de Sauípe faz limites com os municípios de Esplanada, Cardeal da Silva, Alagoinhas e Mata de São João.

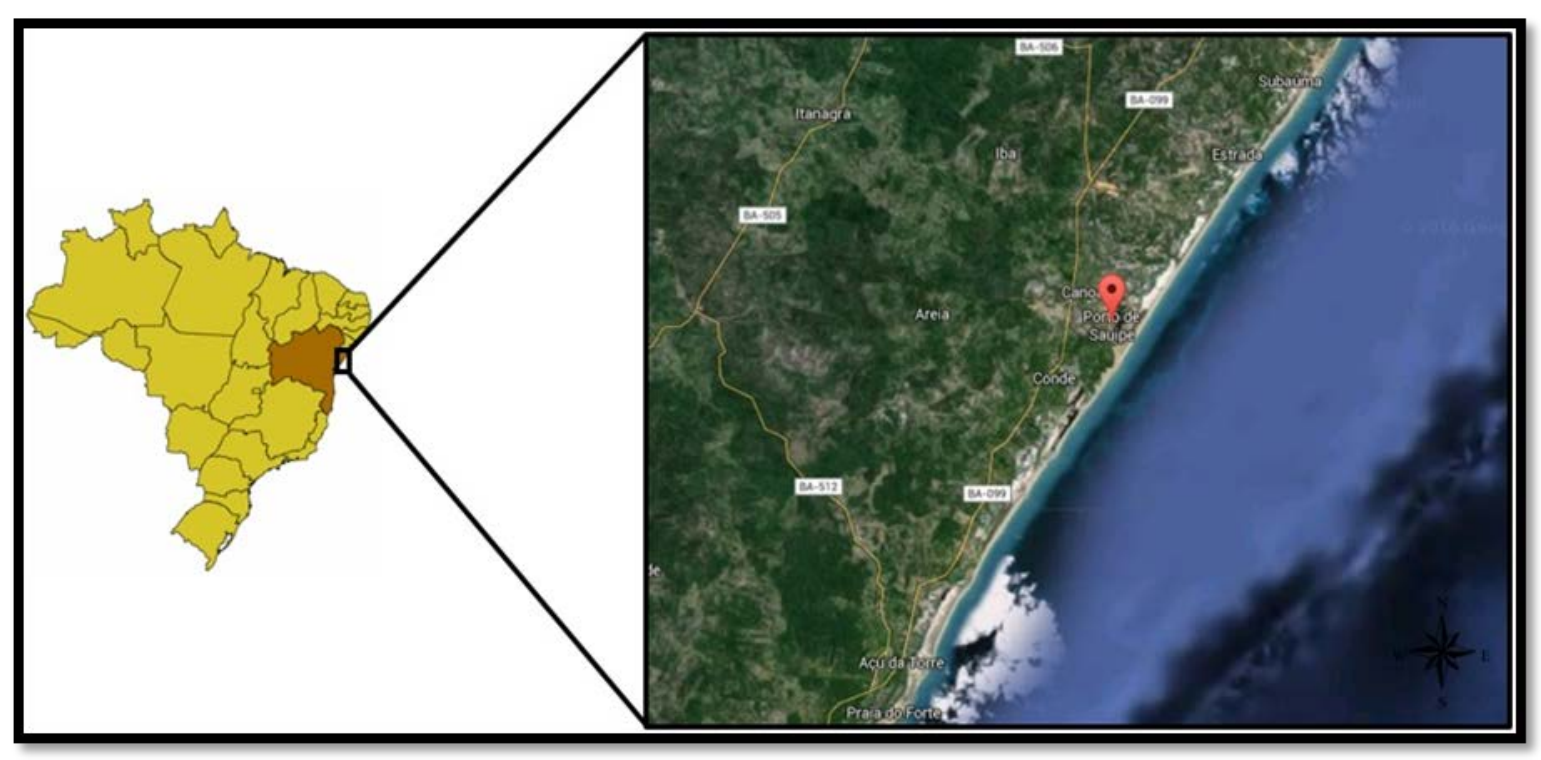

Figura 1. Localização do local de coleta no Porto de Sauípe (Entre Rios, Bahia - Brasil). 2016 Fonte: Google Maps (modificado).

O material foi coletado em uma unidade amostral, sendo coletado de acordo com os métodos utilizados por Campos (2014), onde foram realizados arrastos superficiais com o 
auxílio de uma rede de plâncton cônica (Figura 2A) com malha de $60 \mu \mathrm{m}$ com trinta centímetros de abertura na boca e sessenta e oito centímetros de comprimento, com copo rosqueável de 20 $\mathrm{cm}$. Logo após os arrastos, o material foi acondicionado em uma garrafa (Figura 2B) e para a conservação dos microrganismos foi acrescentado Solução de Transeau, que é composta por seis partes de água destilada, três partes de álcool etílico a $95^{\circ} \mathrm{Gl}$ e uma parte de formaldeído, na proporção 1:1.

No Laboratório de Recursos do Mar e Águas Continentais (LABMARH), da Universidade do Estado da Bahia - UNEB (Campus II, Alagoinhas), o material coletado foi filtrado em papel filtro e concentrado (Figura 2C).

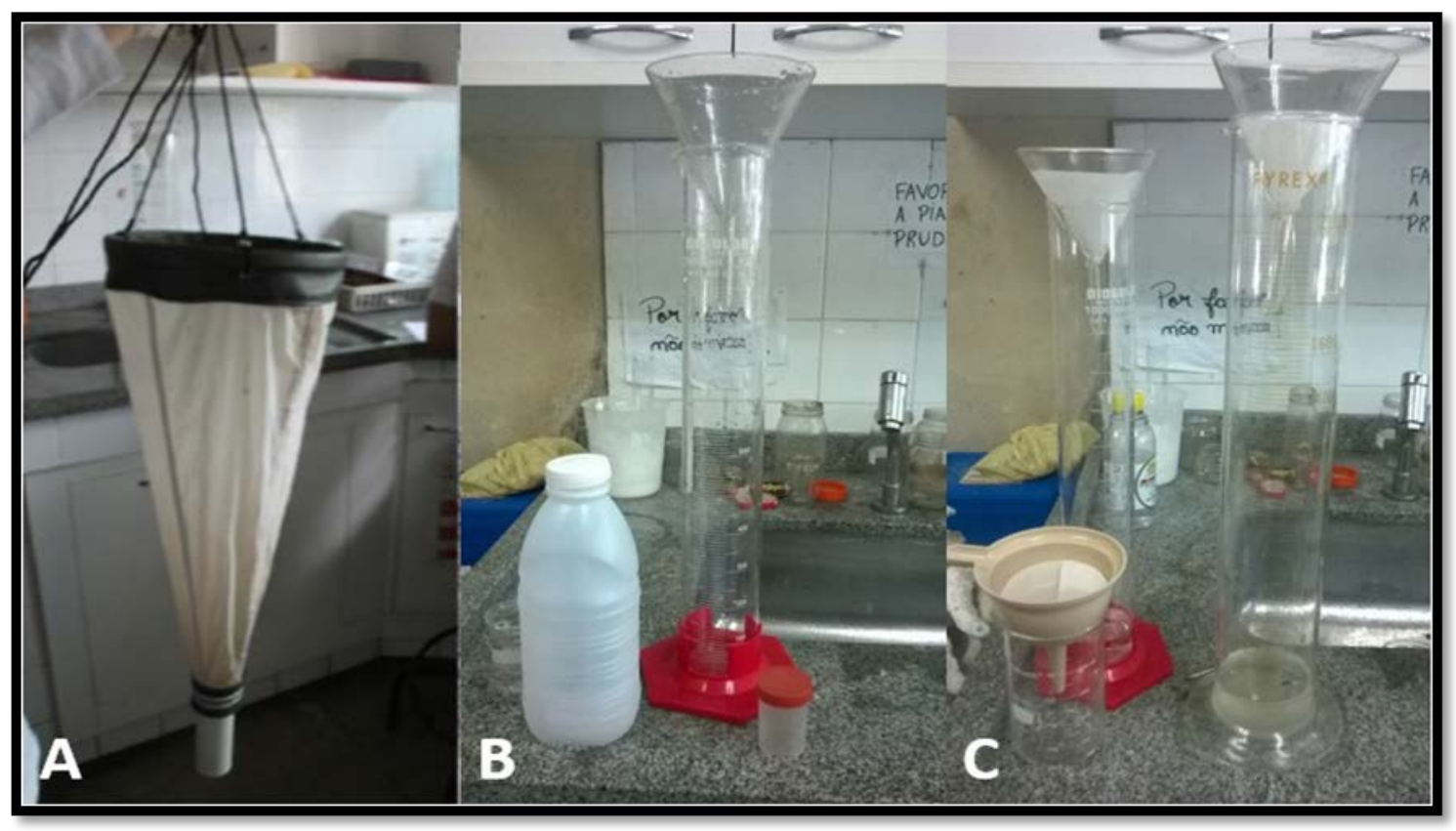

Figura 2. Objetos usados para coleta e filtração do material. A: Rede de plâncton cônica; B: Proveta, funil e recipientes para acondicionar o material antes e depois de serem filtrados; C: Filtração e concentração do material com o uso de papel filtro.

Foram analisados $5 \mathrm{~mL}$ do concentrado de cada amostra ao microscópio óptico (Bioval), usando-se lentes objetivas de até 40x. Desta forma, os espécimes encontrados foram fotografados, identificados e contabilizados. A identificação se deu ao menor nível taxonômico possível, com o auxílio de chaves taxonômicas e obras de especialistas.

Para o tratamento estatístico dos dados foi utilizado a frequência absoluta, obtida a partir do material filtrado e da contagem dos microrganismos ao microscópio óptico. Para a frequência relativa (FR\%) de fito e zooplânctons foi considerado o número de indivíduos de um dos grupos em relação ao total de indivíduos, o resultado foi expresso em porcentagem (\%) e dado pela equação: $f r=f i / n^{*} 100$. Onde, $f r$ é a frequência relativa, $f i$ é a frequência absoluta de indivíduos de um táxon e n é o número total de indivíduos.

Após as identificações e registros, o material analisado nas lâminas foi descartado. Os dados obtidos foram tratados com auxílio do programa Microsoft Office/Excel 2010, para a elaboração das tabelas, gráficos e checklist. A construção dos gráficos de barras foi adotada para demostrar a representatividade dos taxa dentre os resultados obtidos através das frequências. 


\section{RESULTADOS E DISCUSSÃO}

No Estuário do Porto de Sauípe foram identificados um total de 367 indivíduos (Tabela 1), distribuídos em 19 taxa e dois Reinos (Protozoa e Animalia). Dentro do Reino Protozoa, tem-se os representantes identificados do fitoplâncton e zooplâncton, e no Reino Animalia alguns representantes do zooplâncton, parasitas e pequenos invertebrados.

Tabela 1. Checklist, frequências absoluta e relativa (FR\%) da comunidade planctônica coletada no Estuário do Porto de Sauípe (Entre Rios, Bahia - Brasil). 2016.

\begin{tabular}{l|l|l|l}
\hline PROTOZOA & \multicolumn{3}{|l}{} \\
\hline Divisão & Espécie & FA & FR\% \\
\hline Dinoflagelado & Ceratium sp. & 14 & 3,8 \\
\hline \multirow{4}{*}{ Chlorophyta } & Characium oviforme & 50 & 13,6 \\
\cline { 2 - 4 } & Chlorococcum aegyptiacum & 6 & 1,6 \\
\cline { 2 - 4 } & Klebsormidium sp. & 10 & 2,7 \\
\cline { 2 - 4 } & Pseudocharacium americanum & 5 & 1,4 \\
\cline { 2 - 4 } & Scenedesmus ellipticus & 7 & 1,9 \\
\cline { 2 - 4 } & Volvox sp. & 175 & 42,8 \\
\cline { 2 - 4 } & Actinotaenium cucurbita & 2 & 0,5 \\
\hline Turbulinea & Diffugia scalpellum & 15 & 4 \\
\hline Euglenozoa & Euglena sp. & 1 & 0,3 \\
\hline \multirow{2}{*}{ Diatomácea } & Fragilaria sp. & 8 & 2,2 \\
\cline { 2 - 4 } & Hantzschia sp. & 1 & 0,3 \\
\hline Actinopoda (Heliozoa) & Actinophrys sp. & 15 & 4 \\
\hline ANIMALIA & & 3 & 0,8 \\
\hline Nematoda & Ascaris lumbricoides & 9,3 \\
\hline Molusca & Concha de Gastrópode & 9 & 2,4 \\
\hline \multirow{3}{*}{ Crustácea } & Copépoda & 36 & 9,8 \\
\cline { 2 - 4 } & Copépoda Harpacticoida & 1 & 0,3 \\
\cline { 2 - 4 } & Acartia clause & 8 & 2,2 \\
\hline Cordata & Larva de Peixe & 1 & 0,3 \\
\hline N = 367 indivíduos & & \multicolumn{2}{|l}{} \\
\hline & & 3 & \\
\hline
\end{tabular}

O Reino Protozoa (Figuras 3 e 4) foi o grupo de maior representatividade, com uma percentagem de $84,2 \%$ dos indivíduos coletados, sendo que as clorófitas foram destacadas por possuir maior frequência absoluta, representada pelo gênero Volvox sp. O Reino Animalia teve 15,8\% do total amostrado, com os Copépodas como indivíduos mais representativos, tendo uma frequência absoluta de 36, seguindo das conchas de gastrópode com 9. 


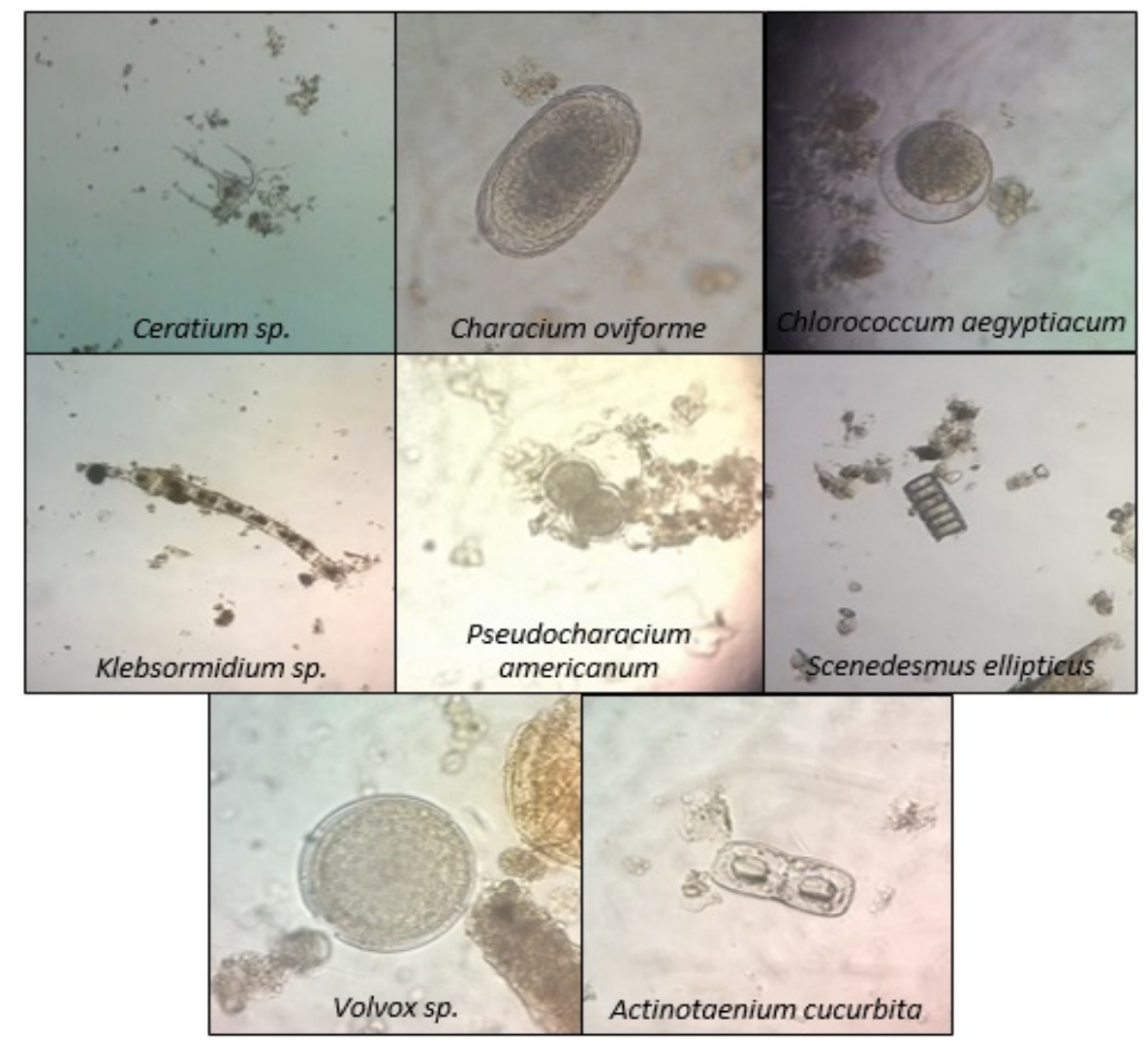

Figura 3. Representantes da comunidade planctônica do Estuário do Rio Sauípe (Entre Rios, Bahia - Brasil). 2016: Dinoflagelado e Chlorophyta.

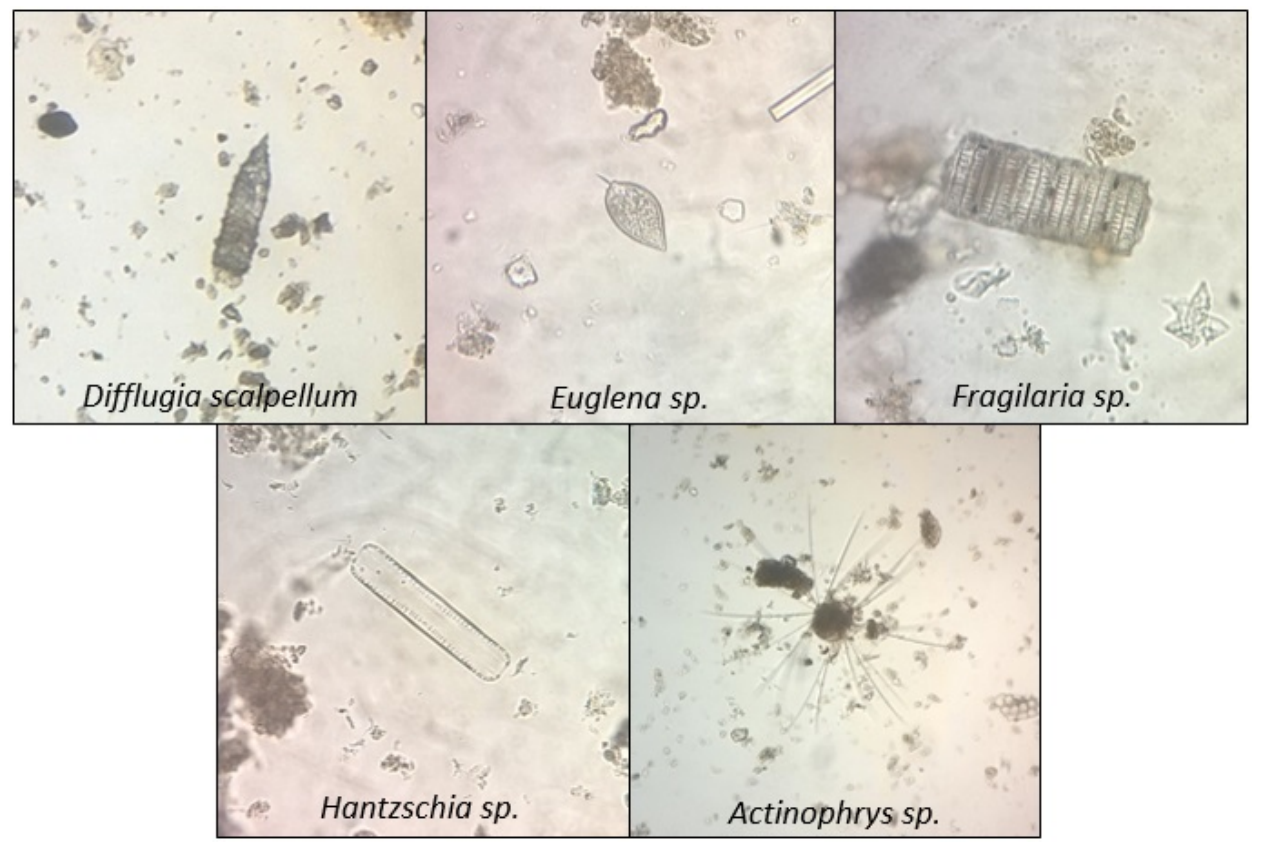

Figura 4. Representantes da comunidade planctônica do Estuário do Rio Sauípe (Entre Rios, Bahia Brasil). 2016: Turbulinea, Euglenozoa, Diatomáceas e Heliozoa. 
Os copépodas apresentam elevado valor na cadeia trófica, pois possuem elevado valor nutricional quando comparados a outras fontes de alimento vivo para larvas de peixes, uma vez que apresentam amplo espectro de tamanho, alta digestibilidade e nutrientes essenciais, permitindo melhor crescimento e desenvolvimento dos organismos cultivados (PUELLO-CRUZ et al., 2009).

Dos indivíduos analisados, a espécie Volvox sp. teve a maior frequência $(42,8 \%)$, seguida de Characium oviforme, com frequência de 13,6\% (Figur 5).

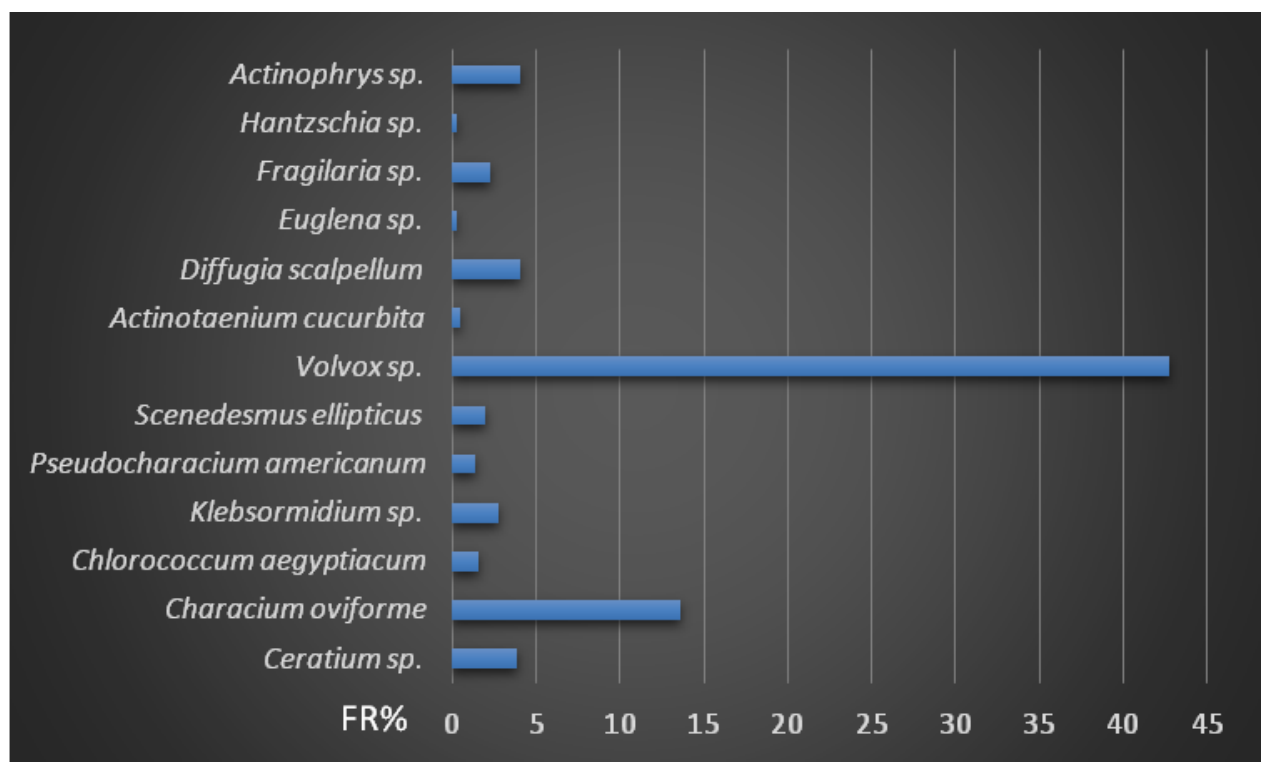

Figura 5. Relação das Frequências relativas (FR\%) do plâncton (Protozoa) do Estuário do Porto de Sauípe (Entre Rios, Bahia - Brasil). 2016.

A elevada frequência do Volvox sp. em áreas de estuário também pode ser observada em outros trabalhos, como se observa em Barbosa (2011) e Silveira Júnior (2012). A grande quantidade de fitoplâncton na amostra coletada é um ponto positivo por representar os organismos produtores primários de ambientes aquáticos. De León e Chalar (2003) apresentam estes organismos como bioindicadores de qualidade de água, caracterizando a estrutura trófica de ambientes aquáticos, composição, abundância, biomassa e diversidade de fitoplâncton.

Os indivíduos com frequência mediana foram Ceratium sp. (Dinoflagelado), Diffugia scalpellum (Turbulinea) e Actinophrys sp. (Heliozoa), com 3,8\%, 4\% e 4\% respectivamente. As menores frequências foram da diatomácea Hantzschia sp. (0,3\%), da Euglena sp. (0,3\%) e da clorófita Actinotaenium cucurbita (0,5\%).

Quanto ao Reino Animalia (Figura 6), foram coletados indivíduos representantes dos taxa Nematoda, Mollusca, Crustacea e Chordata. 


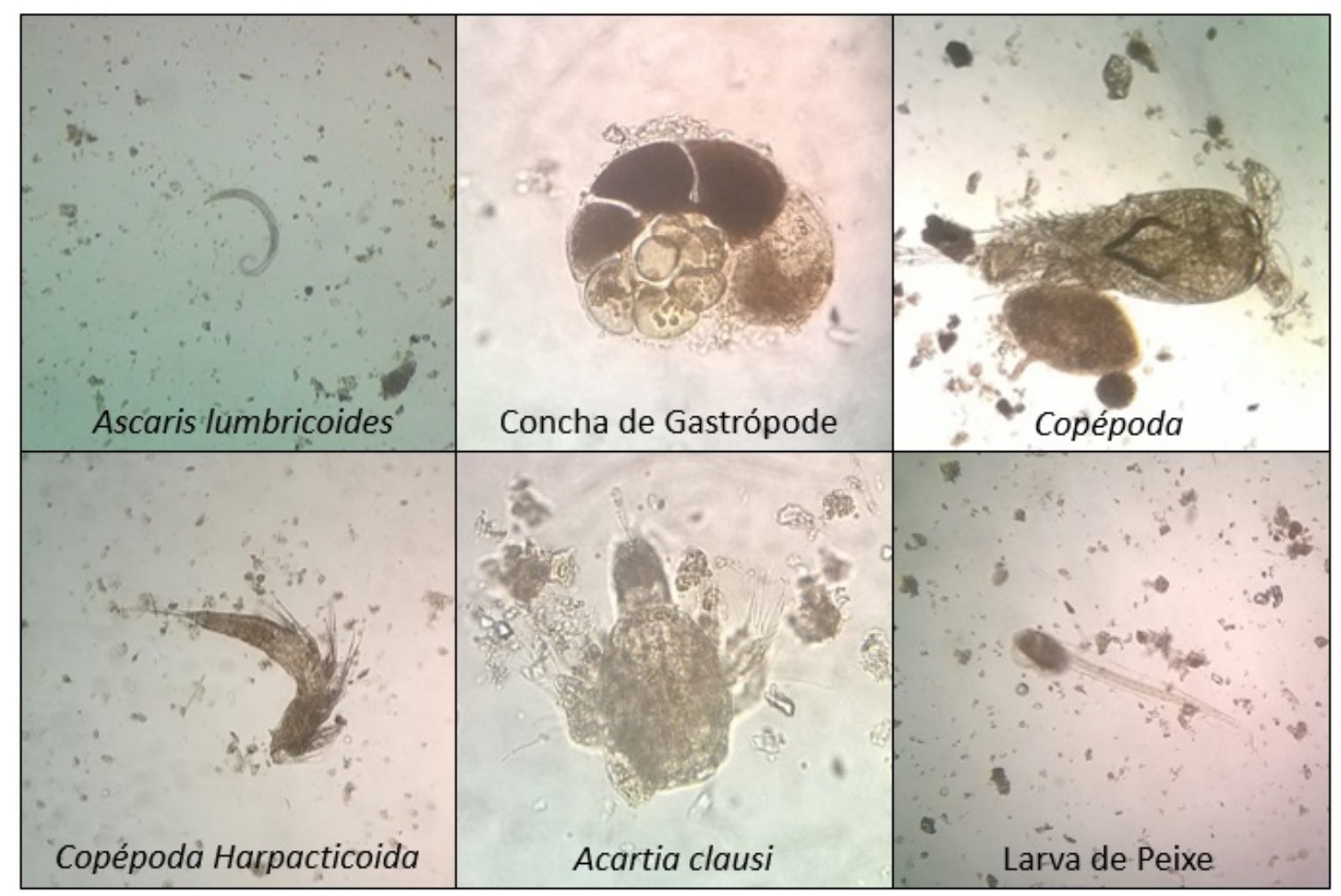

Figura 6. Representantes da comunidade zooplanctônica do estuário do Porto de Sauípe (Entre Rios, Bahia - Brasil) 2016: Nematoda, Molluca, Crustácea e Cordata.

Destes, o mais representativo, com frequência relativa de 9,8\%, foi o crustáceo copépoda (Figura 7), seguido de concha de gastrópode (2,4\%) e Acartia clausi (2,2\%), que é da ordem Calanoida dentro dos copépodas.

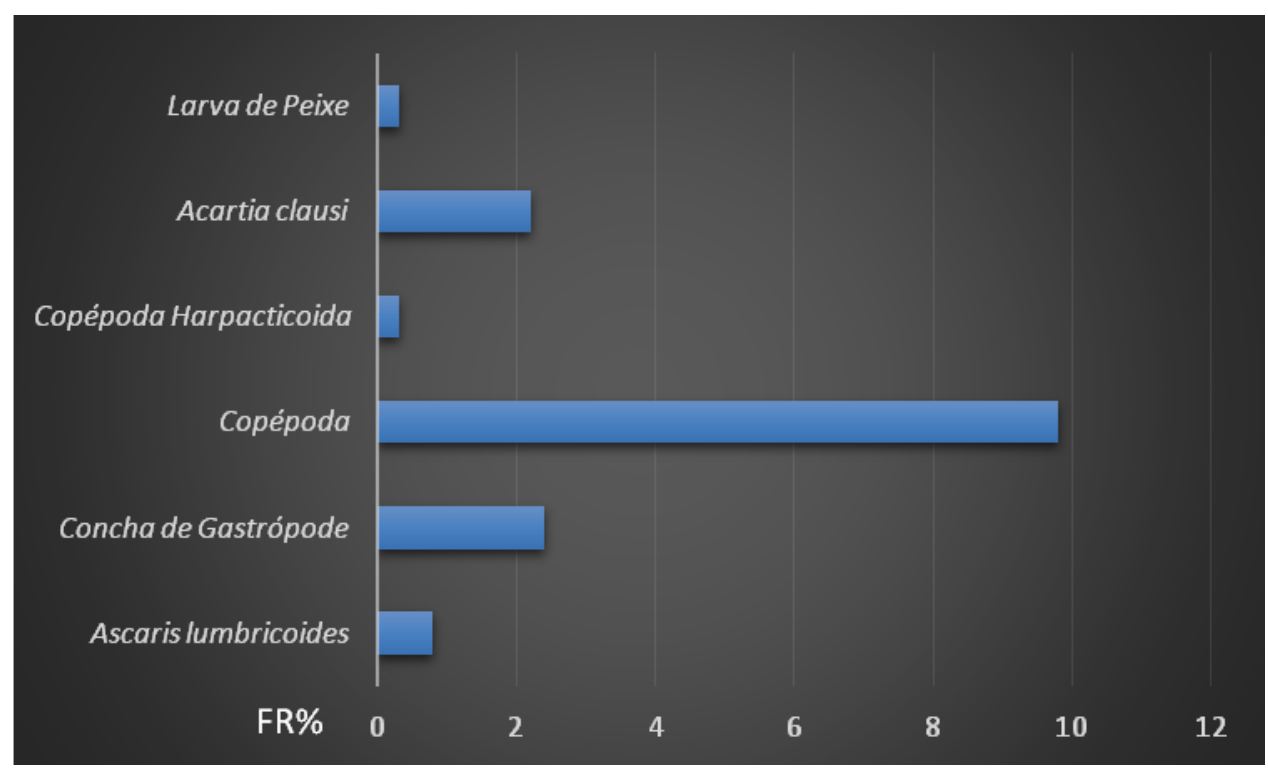

Figura 7. Relação das Frequências relativas (FR\%) do zooplâncton (Animalia) do estuário do rio Sauípe (Entre Rios, Bahia - Brasil). 2016. 
Os menos representativos são o parasita Ascaris lumbricoides (0,8\%), o copépoda da ordem Harpacticoida $(0,3 \%)$ e a larva de peixe $(0,3 \%)$. As larvas de peixe migram para zonas mais protegidas como as margens dos estuários, ficam entre as raízes da vegetação ou deslocam-se para áreas mais profundas. A presença do parasita pode estar relacionada a um certo grau de poluição local, em que Cruz (2014) explana que os helmintos estão associados a práticas de higiene e/ou saneamento básico precários a inexistentes. O Ascaris sp. é um dos gêneros mais resistentes entre os patógenos entéricos e é frequentemente usado como indicador parasitológico (WATSON et al., 1983).

\section{CONCLUSÕES}

Percebe-se uma elevada frequência em representantes do Reino Protozoa, em especial as Clorófitas, o que indica uma boa qualidade do estuário. Quanto aos animais, encontrou-se em maior representatividade os microcrustáceos copépodas, que por sua vez são um dos mais importantes zooplânctons atuantes na cadeia trófica, atuando como elo. No entanto, a presença do parasita Ascaris lumbricoides pode apresentar algum grau de poluição local, mesmo em pouca quantidade como foi registrado na amostra coletada.

\section{REFERÊNCIAS}

BARBOSA, W. F. A. Estrutura da comunidade fitoplanctônica do Estuário do Rio São Francisco (Alagoas/Sergipe, Brasil). Recife: Universidade Federal de Pernambuco. 2011.

CAMPOS, F. L. Caracterização da comunidade zooplactônica do Riacho do Mel, Alagoinhas-BA. Alagoinhas: Universidade do Estado da Bahia, 2014. (Trabalho de Conclusão de curso).

CRUZ, F. V. A. S. Avaliação da microfauna de importância sanitária presente em efluentes de diferentes processos de tratamento de esgotos. Rio de Janeiro: Universidade Federal do Rio de Janeiro, 2014. (Dissertação de Mestrado). Disponível em: <http://dissertacoes.poli.ufrj.br/dissertacoes/dissertpoli1324.pdf>. Acesso em: 23 abr. 2016.

DE LEÓN, L.; CHALAR, G. Abundancia y diversidad del fitoplancton en el Embalse de Salto Grande (Argentina-Uruguay). Ciclo estacional y distribución espacial. Limnetica, v. 22, n. 1/2, p. 103-113, 2003. Disponível em: <http://limno.fcien.edu.uy/pdf/ DE_LEON_CHALAR_2003.pdf>. Acesso em: 23 abr. 2016.

OLIVEIRA, I. B., BICUDO, C. E. M.; MOURA, C. W. N. Euastrum (Desmidiaceae, Zygnematophyceae), na planície litorânea do norte da Bahia, Brasil. Sitientibus, v. 11, p. 62-73, 2011.

PUELLO-CRUZ, A. C.; MEZO-VILLALOBOS, S.; GONZÁLEZ-RODRÍGUEZA, B.; VOLTOLINA, D. Culture of the calanoid copepod Pseudodiaptomus euryhalinus (Johnson 1939) with different microalgal diets. Aquaculture, v. 290, n. 3/4, p. 317-319, 2009. http://dx.doi.org/10.1016/j.aquaculture.2009.02.016

SILVEIRA JÚNIOR, A. M. Composição e biomassa microfitoplanctônica associadas a variáveis físico e químicas em dois transectos da zona estuarina do rio Amazonas (Amapá, Amazônia, Brasil). Macapá: Universidade Federal do Amapá, 2012. (Dissertação de Mestrado). 
WATSON, D. C.; SATCHWELLL, M.; JONES, C. E. A study of the prevalence of parasitic helminth eggs and cysts in sewage sludges disposed to agricultural land: study of eggs and cysts in sewage. Water Pollution Control, p. 285-289, 1983.

YONEDA, N. T. Plâncton. Curitiba: Centro de Estudos do Mar, Universidade Federal do Paraná, 1999. 